\title{
ABOUT THE RATE OF RATIONAL APPROXIMATION OF SOME ANALYTIC FUNCTIONS
}

\author{
A.YA. RADYNO \\ Belorussian State University \\ Skariny av. 4, 220050 Minsk, Belarus
}

\begin{abstract}
The article is devoted to results relating to the theory of rational approximation of an analytic function. Let $f$ be an analytic function on the disk $\{z:|z|<\rho\}, \rho>1$. The rate of decrease of the best approximations $\rho_{n}$ of a function $f$ by the rational functions of order at most $n$ in the uniform metric on the unit disk $E$ with the center $z=0$ is investigated. The theorem connecting the rate of decrease of $\rho_{n}$ with the order $\sigma \geq 0$ of $f$ in the disk $\{z:|z|<\rho\}$ is proved. The proof of this results is based on an analysis of behavior of the singular numbers of the Hankel operator constructed from the function $f$.
\end{abstract}

\section{INTRODUCTION}

Let $E=\{z:|z| \leq 1\}$, and $f$ is an analytic function on the disk $\{z:|z|<$ $\rho\}, \rho>1$. For any nonnegative integer $n$ denote by $\mathcal{R}_{n}$ a class of rational functions with complex coefficients of order at most $n$. For each $n=0,1,2, \ldots$ we define the number $\rho_{n}$ by the next formula:

$$
\rho_{n}=\rho_{n}(f ; E)=\inf _{r \in \mathcal{R}_{n}} \max _{z \in E}|f(z)-r(z)| .
$$

It is well known that (see [1], [2])

$$
\limsup _{n \rightarrow \infty}\left(\rho_{1} \rho_{2} \ldots \rho_{n}\right)^{1 / n^{2}} \leq 1 / \rho
$$

This inequality yields

$$
\liminf _{n \rightarrow \infty} \rho_{n}^{1 / n} \leq 1 / \rho^{2},
$$

which confirms Gonchar's conjecture.

Definition 1. We define an order $\sigma$ of the function $f$ in the disk $\{z:|z|<$ 
$\rho\}, \rho>1$, by the formula:

$$
\sigma=\sigma(f)=\limsup _{r \rightarrow \rho} \ln ^{+} \ln ^{+} \max _{|z|=r}|f(z)| / \ln (1 /(1-r / \rho)),
$$

where $\ln ^{+} x=\max (0, \ln x)$ for $x \geq 0$.

An investigation of behavior of singular numbers of Hankel operator constructed from function to be approximated allows to specify mentioned estimates (1) and (2) for holomorphic functions having the finite order in the disk $\{z:|z|<\rho\}$.

THEOREM 1. Let $f$ be holomorphic on the open disk $\{z:|z|<\rho\}, \rho>1$, and let $\sigma \geq 0$ be an order of the function $f$ in $\{z:|z|<\rho\}$. Then

$$
\limsup _{n \rightarrow \infty} \frac{\ln ^{+} \ln ^{+}\left(\rho_{0} \rho_{1} \cdots \rho_{n} \cdot \rho^{n(n+1)}\right)}{\ln n} \leq 1+\frac{\sigma}{\sigma+1} .
$$

We give some corollaries. By using the inequalities $\rho_{n} \leq \rho_{n-1} \leq \cdots \leq \rho_{0}$, we get the following upper estimate for $\lim \sup \ln ^{+} \ln ^{+}\left(\rho_{n} \rho^{n}\right) / \ln n$.

$$
n \rightarrow \infty
$$

Corollary 2.

$$
\limsup _{n \rightarrow \infty} \frac{\ln ^{+} \ln ^{+}\left(\rho_{n} \rho^{n}\right)}{\ln n} \leq \frac{\sigma}{\sigma+1}
$$

Theorem 1 also gives us an upper estimate of $\liminf _{n \rightarrow \infty} \ln ^{+} \ln ^{+}\left(\rho_{n} \rho^{2 n}\right) / \ln n$.

Corollary 3.

$$
\liminf _{n \rightarrow \infty} \frac{\ln ^{+} \ln ^{+}\left(\rho_{n} \rho^{2 n}\right)}{\ln n} \leq \frac{\sigma}{\sigma+1}
$$

\section{INVESTIGATION OF THE HANKEL OPERATOR}

In this section we present results needed below from the theory of the Hankel operator.

Let $G$ be the disk of radius $r, 0<r<\rho$, with center $z=0$, and $\Gamma$ is a boundary of $G$. Denote by $H_{2}(G)$ the Hardy class of analytic functions in the disk $G$. Each function in the class $H_{2}(G)$ has limit values alone paths nontangential to $\Gamma$ at almost all points $\xi \in \Gamma$. The function determined on $\Gamma$ by this limit values belongs to the space $L_{2}(\Gamma)$. The functions in the class $H_{2}(G)$ are uniquely determined by their limit values and in this sense we will write that $H_{2}(G) \subset L_{2}(\Gamma)$. We represent the space $L_{2}(\Gamma)$ as a direct sum $L_{2}(\Gamma)=H_{2}(G) \oplus H_{2}(G)^{\perp}$ of subspaces, where $H_{2}(G)^{\perp}$ is a orthogonal complement of $H_{2}(G)$ in $L_{2}(\Gamma)$. 
The Hankel operator $A_{f}: H_{2}(G) \rightarrow H_{2}^{\perp}(G)$ is defined as follows. For any function $g \in H_{2}(G)$ let $A_{f} g=\mathbb{P}_{-}(g f)$, where $\mathbb{P}_{-}$is an orthogonal projection of $L_{2}(\Gamma)$ onto $H_{2}(G)^{\perp}$. It is not hard to see that $A_{f}$ is a compact operator.

Denote by $\left\{s_{k}\right\}, k=0,1,2, \ldots$, the sequence of singular numbers (counting multiplicity) of the operator $A_{f}\left(s_{k}\right.$ is an eigenvalue of the operator $\left(A_{f}^{*} A_{f}\right)^{1 / 2}$, where $A_{f}^{*}: H_{2}(G)^{\perp} \rightarrow H_{2}(G)$ is the adjoint operator of $A_{f}$. Assume that $s_{0} \geq s_{1} \geq s_{2} \geq \ldots$ (for more details about singular numbers see [4]).

There exist orthonormal systems of eigenfunctions of the operator $\left(A_{f}^{*} A_{f}\right)^{1 / 2}$ corresponding to the sequence of singular numbers $\left\{s_{n}\right\}, n \geq 0,\left\{q_{n}\right\},\left\{\alpha_{n}\right\}$, such that the following formula for the product of singular numbers is valid:

$$
s_{0} s_{1} \cdots s_{k}=\left|\int_{\Gamma}\left(q_{i} \alpha_{j} f\right)(\xi) d \xi\right|_{i, j=0}^{k}, \quad k \geq 0
$$

where the right-hand side is a determinant of order $k+1$.

For any nonnegative integer $n$ let $\mathcal{M}_{n}(G)$ be the class of functions representable in the form $h=p / q$, where $p \in E_{\infty}(G)$ and $q$ is a polynomial of degree at most $n, q \not \equiv 0$.

Let $\Delta_{n}=\Delta_{n}(f, G)$ be the best approximation of $f$ in the space $L_{\infty}(\Gamma)$ in the class $\mathcal{M}_{n}(G)$ :

$$
\Delta_{n}=\Delta_{n}(f ; G)=\inf _{h \in \mathcal{M}_{n}(G)}\|f-h\|_{\infty}
$$

By the Adamyan-Arov-Kreı̆n theorem for all integers $n \geq 0$ the following equalities are valid (see [6] and [3])

$$
\Delta_{n}=s_{n}
$$

\section{THE PROOF OF THEOREM 1}

Before proving Theorem 1 we remark that we can rewrite the formula (3) for the order of the function $f$ in the disk $\{z:|z|<\rho\}$ in another form.

Let $F=\{z:|z| \geq \rho\}$. Denote by $w_{1}(z)=\ln |z| / \ln \rho$ the solution of the Dirichlet problem in the domain $\overline{\mathbf{C}} \backslash(E \cup F)=\{z: 1<|z|<\rho\}$ with respect to boundary data equal to 1 on $\partial F$ and to 0 on $\partial E$. For $0<\varepsilon<1$ we designate $\gamma_{1}(\varepsilon)=\left\{z: w_{1}(z)=\varepsilon\right\}$.

It is not hard to see that the following formula for the order of the function $f$ in the domain $\overline{\mathbf{C}} \backslash F=\{z:|z|<\rho\}$ is valid:

$$
\sigma=\limsup _{\varepsilon \rightarrow 1} \frac{\ln ^{+} \ln ^{+}\|f\|_{\gamma_{1}(\varepsilon)}}{\ln (1 /(1-\varepsilon))}
$$

where $\|f\|_{\gamma_{1}(\varepsilon)}=\max _{z \in \gamma_{1}(\varepsilon)}|f(z)|$. 
Let $E_{1}=\{\xi:|\xi| \geq 1\}$ and $F_{1}=\{\xi:|\xi| \leq 1 / \rho\}$ be the preimages of $E$ and $F$ under the mapping $z=1 / \xi$. Let $f_{1}(\xi)=f(1 / \xi)$.

We mention that the equality

$$
\rho_{n}(f ; E)=\rho_{n}\left(f_{1} ; E_{1}\right)
$$

holds for all nonnegative integer $n$, where

$$
\rho_{n}\left(f_{1} ; E_{1}\right)=\inf _{r \in \mathcal{R}_{n}}\left\|f_{1}-r\right\|_{E_{1}}
$$

Namely, for this situation we prove that

$$
\limsup _{n \rightarrow \infty} \frac{\ln ^{+} \ln ^{+}\left(\rho_{0}^{*} \rho_{1}^{*} \cdots \rho_{n}^{*} \rho^{n(n+1)}\right)}{\ln n} \leq 1+\frac{\sigma}{\sigma+1},
$$

where $\rho_{n}^{*}=\rho_{n}\left(f_{1} ; E_{1}\right)$. Hence, by $(9)$, we get (4).

We introduce the necessary notation. Let $w(z)$ be the solution of the Dirichlet problem constructed the domains making up the domain $\overline{\mathbf{C}} \backslash\left(E_{1} \cup F_{1}\right)=$ $\{z: 1 / \rho<|\xi|<1\}$ with respect to boundary data equal to 1 on $\partial F_{1}$ and to 0 on $\partial E_{1}$. It will be assumed that $w(z)$ is extended by continuity to $\overline{\mathbf{C}}$ : $w(z)=1$ for $z \in F_{1}$, and $w(z)=0$ for $z \in E_{1}$. For an arbitrary number $\varepsilon$ with $0<\varepsilon<1$ let $\gamma(\varepsilon)=\{z: w(z)=\varepsilon\}$ and $G(\varepsilon)=\{z: w(z)>\varepsilon\}$.

Before continuing with the proof of the theorem we note that the capacity $C\left(E_{1}, F_{1}\right)$ of the condenser $\left(E_{1}, F_{1}\right)$ satisfies the following relations:

$$
C\left(E_{1}, F_{1}\right)=\frac{1}{\ln \rho}
$$

and

$$
C\left(\gamma\left(\varepsilon_{1}\right), \gamma\left(\varepsilon_{2}\right)\right)=\frac{C\left(E_{1}, F_{1}\right)}{\varepsilon_{1}-\varepsilon_{2}} \quad \text { for } \quad 0<\varepsilon_{2}<\varepsilon_{1}<1 .
$$

Fix an arbitrary number $\sigma^{\prime}>\sigma$ and $0<\alpha<1 / 2$. Let $\varepsilon_{3, n}=\varepsilon_{2, n} / 2=$ $\alpha / 2 n, n=1,2, \ldots, \varepsilon_{3,0}=\varepsilon_{2,0} / 2=\alpha$. Here it is assumed that $\gamma\left(\varepsilon_{3, n}\right)$ is positively oriented with respect to $G\left(\varepsilon_{3, n}\right)$.

In this subsection we prove the inequality

$$
\Delta_{0} \Delta_{1} \cdots \Delta_{n} \rho^{n(n+1)} \leq(n+1) ! c_{0}^{n} n^{5 n} \exp \left(c_{1} n^{1+\sigma^{\prime} /\left(\sigma^{\prime}+1\right)}\right),
$$

where $c_{0}$ and $c_{1}$ are positive constants and

$$
\Delta_{n}=\Delta_{n}\left(f_{1} ; G\left(\varepsilon_{3, n}\right)\right)=\inf _{h}\left\|f_{1}-h\right\|_{\infty}, \quad j=0,1, \ldots, n
$$


is the best approximation of $f_{1}$ in $L_{\infty}\left(\partial G\left(\varepsilon_{3, n}\right)\right)$ in the class $\mathcal{M}_{n}\left(G\left(\varepsilon_{3, n}\right)\right)$.

For this it suffices to show (see the equalities (7)) that

$$
s_{0} s_{1} \cdots s_{n} \rho^{n(n+1)} \leq(n+1) ! c_{0}^{n} n^{5 n} \exp \left(c_{1} n^{1+\sigma^{\prime} /\left(\sigma^{\prime}+1\right)}\right),
$$

where $\left\{s_{k}\right\}, s_{k}=s_{k}\left(f_{1} ; G\left(\varepsilon_{3, n}\right)\right), k=0,1,2, \ldots$, is the sequence of singular numbers of the Hankel operator $A_{f_{1}}: H_{2}\left(G\left(\varepsilon_{3, n}\right)\right) \rightarrow H_{2}^{\perp}\left(G\left(\varepsilon_{3, n}\right)\right)$, constructed from the function $f_{1}$.

It is not difficult to pass from the estimate (13) to (10), therefore we now restrict ourselves to proving the inequality (14).

We choose and fix a positive integer $n$ such that the contour $\gamma\left(\varepsilon_{1, n}\right)$,

$$
\varepsilon_{1, n}=1-\left(C\left(E_{1}, F_{1}\right) \sigma^{\prime} / n\right)^{1 /\left(\sigma^{\prime}+1\right)},
$$

belongs to the domain $G\left(\varepsilon_{2, n}\right)$. It will be assumed that $\gamma\left(\varepsilon_{1, n}\right)$ is positively oriented with respect to $G\left(\varepsilon_{1, n}\right)$.

Let us use the formula (6) with $k=n$. Since the functions $q_{i}, \alpha_{j}, i, j=$ $0,1,2, \ldots$, belong to $H_{2}\left(G\left(\varepsilon_{3, n}\right)\right)$ and $f_{1}$ is holomorphic on $\overline{\mathbf{C}} \backslash F_{1}$, the relation

$$
s_{0} s_{1} \cdots s_{n}=\left|\int_{\gamma\left(\varepsilon_{1, n}\right)}\left(q_{i} \alpha_{j} f_{1}\right)(\xi) d \xi\right|_{i, j=0}^{n}
$$

can be written for the product of singular numbers. From the last relation

$$
\begin{aligned}
(n+1) ! \prod_{i=0}^{n} s_{i}= & \int_{\gamma\left(\varepsilon_{1, n}\right)} \cdots \int_{\gamma\left(\varepsilon_{1, n}\right)} f_{1}\left(\xi_{0}\right) \cdots f_{1}\left(\xi_{n}\right) \\
& \times B_{1}\left(\xi_{0}, \ldots, \xi_{n}\right) B_{2}\left(\xi_{0}, \ldots, \xi_{n}\right) d \xi_{0} \cdots d \xi_{n}
\end{aligned}
$$

where

$$
B_{1}\left(\xi_{0}, \xi_{1}, \ldots, \xi_{n}\right)=\left|\alpha_{i}\left(\xi_{j}\right)\right|_{i, j=0}^{n}
$$

and

$$
B_{2}\left(\xi_{0}, \xi_{1}, \ldots, \xi_{n}\right)=\left|q_{i}\left(\xi_{j}\right)\right|_{i, j=0}^{n} .
$$

We estimate determinants $B_{1}$ and $B_{2}$.

By the Cauchy formula,

$$
\alpha_{j}^{2}(\xi)=\frac{1}{2 \pi i} \int_{\gamma\left(\varepsilon_{3, n}\right)} \frac{\alpha_{j}^{2}(t)}{t-\xi} d t, \xi \in \gamma\left(\varepsilon_{2, n}\right), j=0,1,2, \ldots
$$

Since the norm $\alpha_{j}, j=0,1,2, \ldots$, in the space $L_{2}\left(\gamma\left(\varepsilon_{3, n}\right)\right)$ is 1 , it follows from the last formula that

$$
\max _{\xi \in \gamma\left(\varepsilon_{2, n}\right)}\left|\alpha_{j}^{2}(\xi)\right| \leq C / \operatorname{dist}\left(\gamma\left(\varepsilon_{3, n}\right), \gamma\left(\varepsilon_{2, n}\right)\right), \quad j=0,1,2, \ldots
$$


(here and in what follows $C, C_{1}, C_{2}, \ldots$ will denote positive quantities not depending on $n$ ). Similarly, since the norm $q_{j}, j=0,1,2, \ldots$, in the space $L_{2}\left(\gamma\left(\varepsilon_{3, n}\right)\right)$ is 1 , it follows that

$$
\max _{\xi \in \gamma\left(\varepsilon_{2, n}\right)}\left|q_{j}^{2}(\xi)\right| \leq C / \operatorname{dist}\left(\gamma\left(\varepsilon_{3, n}\right), \gamma\left(\varepsilon_{2, n}\right)\right), \quad j=0,1,2, \ldots
$$

Using the inequalities (19), (20) and $\operatorname{dist}\left(\gamma\left(\varepsilon_{3, n}\right), \gamma\left(\varepsilon_{2, n}\right)\right) \geq C_{1} / n$, we can write

$$
\max _{\xi_{i} \in \gamma\left(\varepsilon_{2, n}\right)}\left|B_{1}\left(\xi_{0}, \ldots, \xi_{n}\right) B_{2}\left(\xi_{0}, \ldots, \xi_{n}\right)\right| \leq((n+1) !)^{2} n^{n+1} C_{2}^{n+1}
$$

Denote by $g(z, \xi)$ the Green's function of the domain $G\left(\varepsilon_{2, n}\right)$ with singularity at the point $\xi \in G\left(\varepsilon_{2, n}\right)$. We estimate the product $B_{1} B_{2}$ in the case when the variables $\xi_{i}, i=0, \ldots, n$, belong to $\gamma\left(\varepsilon_{1, n}\right)$. We use (17) and (18), obtaining

$$
\begin{aligned}
D\left(\xi_{0}, \ldots, \xi_{n}\right) & =B_{1}\left(\xi_{0}, \ldots, \xi_{n}\right) B_{2}\left(\xi_{0}, \ldots, \xi_{n}\right) \\
& =\prod_{0 \leq i<j \leq n}\left(\xi_{i}-\xi_{j}\right)^{2} \cdot \Psi\left(\xi_{0}, \ldots, \xi_{n}\right),
\end{aligned}
$$

where $\Psi\left(\xi_{0}, \ldots, \xi_{n}\right)$ is a holomorphic function of $n+1$ complex variables in the domain $G\left(\varepsilon_{2, n}\right) \times \cdots \times G\left(\varepsilon_{2, n}\right)(n+1$ factors in the Cartesian product).

Let us consider the function

$$
\ln \left|D\left(\xi_{0}, \xi_{1}, \ldots, \xi_{n}\right)\right|+2 \sum_{0 \leq i<j \leq n} g\left(\xi_{i}, \xi_{j}\right)
$$

We underscore that the given function is subharmonic in the domain $G\left(\varepsilon_{2, n}\right)$ with respect to the variable $\xi_{i}, i=0, \ldots, n$, when the remaining variables $\xi_{j} \in G\left(\varepsilon_{2, n}\right), j \neq i, j \in\{0,1, \ldots, n\}$, are fixed.

We use the maximum principle for subharmonic functions successively with respect to each variable, together with (21) and (22), obtaining

$$
\ln \left|D\left(\xi_{0}, \xi_{1}, \ldots, \xi_{n}\right)\right|+2 \sum_{0 \leq i<j \leq n} g\left(\xi_{i}, \xi_{j}\right) \leq \ln \left(((n+1) !)^{2} n^{n+1} C_{3}^{n+1}\right),
$$

where $\xi_{i} \in \gamma\left(\varepsilon_{1, n}\right), i=0,1, \ldots, n$.

By the formula for a product of singular numbers (see (16)), this gives us the inequality

$$
\prod_{i=0}^{n} s_{i} \leq(n+1) ! C_{4}^{n} n^{n+1}\left(\left\|f_{1}\right\|_{\gamma\left(\varepsilon_{1, n}\right)}\right)^{n+1} e^{-w_{n}}
$$


where

$$
w_{n}=\min _{\xi_{i} \in \gamma\left(\varepsilon_{1, n}\right)}\left(2 \sum_{0 \leq i<j \leq n} g\left(\xi_{i}, \xi_{j}\right)\right) .
$$

Let us estimate the right-hand side of the inequality (24). We note first that since $\omega(\xi)=\omega_{1}(1 / \xi)$ then, by $(8)$,

$$
\sigma=\lim _{\varepsilon \rightarrow 1} \frac{\ln ^{+} \ln ^{+}|| f_{1} \|_{\gamma(\varepsilon)}}{\ln (1 /(1-\varepsilon))}
$$

and, by (15), $f_{1}$ admits the upper estimate

$$
\left\|f_{1}\right\|_{\gamma\left(\varepsilon_{1, n}\right)} \leq \exp \left(\left(n / C\left(E_{1}, F_{1}\right) \sigma^{\prime}\right)^{\sigma^{\prime} /\left(\sigma^{\prime}+1\right)}\right) .
$$

We next use the following relation (see [5])

$$
-w_{n} \leq-\frac{n(n+1)}{C\left(\gamma\left(\varepsilon_{1, n}\right), \gamma\left(\varepsilon_{2, n}\right)\right)}+k(n+1)+(n+1) \ln (n+1),
$$

where $k>0$ is a positive constant. This, by (12), implies

$$
-w_{n} \leq \frac{-n(n+1)\left(\varepsilon_{1, n}-\varepsilon_{2, n}\right)}{C\left(E_{1}, F_{1}\right)}+k(n+1)+(n+1) \ln (n+1) .
$$

From this, since

$$
\varepsilon_{1, n}=1-\left(C\left(E_{1}, F_{1}\right) \sigma^{\prime} / n\right)^{1 / \sigma^{\prime}+1}, \quad \varepsilon_{2, n}=\alpha / n, \quad n=1,2, \ldots,
$$

and, by (11), we get

$$
\begin{aligned}
\left(\prod_{i=0}^{n} s_{i}\right) \rho^{n(n+1)} \leq & (n+1) ! C_{4}^{n} n^{n+1} \exp \left((n+1)\left(n / C\left(E_{1}, F_{1}\right) \sigma^{\prime}\right)^{\sigma^{\prime} /\left(\sigma^{\prime}+1\right)}\right. \\
+ & n(n+1)\left(\left(C\left(E_{1}, F_{1}\right) \sigma^{\prime} / n\right)^{1 /\left(\sigma^{\prime}+1\right)}+\alpha / n\right) / C\left(E_{1}, F_{1}\right) \\
+ & k(n+1)+(n+1) \ln (n+1))
\end{aligned}
$$

and

$$
\begin{aligned}
\left(\prod_{i=0}^{n} s_{i}\right) \rho^{n(n+1)} & \leq(n+1) ! C_{5}^{n} n^{5 n} \exp \left((n+1)\left(n / C\left(E_{1}, F_{1}\right) \sigma^{\prime}\right)^{\sigma^{\prime} /\left(\sigma^{\prime}+1\right)}\right. \\
& \left.+n(n+1)\left(C\left(E_{1}, F_{1}\right) \sigma^{\prime} / n\right)^{1 / \sigma+1} / C\left(E_{1}, F_{1}\right)\right) \\
& =(n+1) ! C_{6}^{n} n^{5 n} \exp \left(C_{7} n^{1+\sigma^{\prime} /\left(\sigma^{\prime}+1\right)}\right) .
\end{aligned}
$$


As mentioned above, the relation (13) is thereby obtained.

We now show how to use the estimate (13) to get the inequality (10).

Fix positive integer $n$. For an arbitrary function $h$ representable in the form $h=p / q$, where $p \in E_{\infty}\left(G\left(\varepsilon_{3, n}\right)\right), q$ is a polynomial of degree at most $n$, with zeros outside $\gamma\left(\varepsilon_{3, n}\right), q \not \equiv 0$, we have by the Cauchy formula

$$
\left(r^{\prime}-f_{1}\right)(z)+f_{1}(\infty)=\frac{1}{2 \pi i} \int_{\gamma\left(\varepsilon_{3, n}\right)} \frac{\left(f_{1}-h\right)(\xi) d \xi}{\xi-z}, \quad z \in E_{1}
$$

where $r^{\prime}$ is the sum of the principal parts of $h$ corresponding to poles of $h$ laying in $G\left(\varepsilon_{3, n}\right)$.

We estimate the integral in (27), getting

$$
\left\|f_{1}-f_{1}(\infty)-r^{\prime}\right\|_{E_{1}} \leq C_{6}\left\|f_{1}-h\right\|_{\infty} / \operatorname{dist}\left(E_{1}, \gamma\left(\varepsilon_{3, n}\right)\right),
$$

where the positive quantity $C_{6}$ is independent of $h, n$, and $j$, and $\|\cdot\|_{\infty}$ is the norm in the space $L_{\infty}\left(\gamma\left(\varepsilon_{3, n}\right)\right)$.

Using now the definition of the quantity $\rho_{n}$ and the fact that the rational function $r^{\prime}+f_{1}(\infty)$ belongs to the class $\mathcal{R}_{n}$, we have from (28) the estimate

$$
\rho_{n}^{*} \leq C_{6}|| f_{1}-h \|_{\infty} / \operatorname{dist}\left(E_{1}, \gamma\left(\varepsilon_{3, n}\right)\right)
$$

Next, since $h$ is an arbitrary function in $\mathcal{M}_{n}\left(G\left(\varepsilon_{3, n}\right)\right)$ and dist $\left(E_{1}\right.$, $\left.\gamma\left(\varepsilon_{3, n}\right)\right) \geq C / n$

$$
\rho_{n}^{*} \leq C_{7} n \inf _{h \in \mathcal{M}_{n}\left(G\left(\varepsilon_{3, n}\right)\right)}\left\|f_{1}-h\right\|_{\infty}=C_{7} n \Delta_{n}\left(f_{1} ; G\left(\varepsilon_{3, n}\right)\right) .
$$

We now use the relation (13) to get

$$
\limsup _{n \rightarrow \infty} \frac{\ln ^{+} \ln ^{+}\left(\rho_{0}^{*} \rho_{1}^{*} \cdots \rho_{n}^{*}\right) \rho^{n(n+1)}}{\ln n} \leq 1+\frac{\sigma^{\prime}}{\sigma^{\prime}+1} .
$$

It remains to let $\sigma^{\prime}$ tend to $\sigma$, and get the required relation (10).

\section{THE PROOF OF COROLLARY 3.}

The proof of Corollary 3 is given by contradiction. Let

$$
\liminf _{n \rightarrow \infty} \frac{\ln ^{+} \ln ^{+}\left(\rho_{n} \rho^{2 n}\right)}{\ln n} \geq \frac{\lambda \sigma}{\sigma+1},
$$


where $\lambda>1$. Then by the relation

$$
\lim _{n \rightarrow \infty} \frac{\sum_{k=0}^{n} k^{\sigma /(\sigma+1)}}{n^{1+\sigma /(\sigma+1)}}=\frac{1}{1+\sigma /(\sigma+1)}
$$

we get

$$
\liminf _{n \rightarrow \infty} \frac{\ln ^{+} \ln ^{+}\left(\rho_{1} \rho_{2} \cdots \rho_{n} \rho^{n(n+1)}\right)}{\ln n} \geq \lambda\left(1+\frac{\sigma}{\sigma+1}\right)>1+\frac{\sigma}{\sigma+1},
$$

which contradicts the inequality (4).

\section{REFERENCES}

[1] Parfenov O.G. //Math. trans., 131 (1986), P. 501 - 518 (in Russian).

[2] Prokhorov V.A.//Math. trans. 184, No 2 (1993), P. 3 - 32, (in Russian).

[3] Prokhorov V.A. //Math. trans. 184, No 1, (1993), P. 89 - 104, (in Russian) .

[4] Gohberg I.T., Krein M.G. Introduction in the theory of the non-self-adjoint operators in the Gilbert's space, Nauka, Moskow, (1976), (in Russian).

[5] Menke K.// Complex Variables Theory Appl., 2 (1983), P. 165 - 175.

[6] Adamyan V. M., Arov D. Z., Krein M. G. //Math. trans., 86 (1971), P. 34 - 75, (in Russian). 